\title{
Estudiantes extranjeros/as en la representación de los docentes en una escuela de Santiago: elementos para una educación intercultural
}

\author{
Pablo Segovia Lagos \\ Universidad Mayor, Santiago, Chile \\ Email: pablo.segovia@umayor.cl \\ Bibiana Rendón Zapata \\ Universidad Mayor, Santiago, Chile \\ Email: bibiana.rendon@mayor.cl
}

\begin{abstract}
Resumen: El artículo aborda la percepción docente en torno a estudiantes migrantes en una escuela pública de Santiago de Chile. Es un estudio de corte cuantitativo, descriptivo correlacional con una muestra conformada por 16 docentes. La recolección de datos se realizó a través de un instrumento ad hoc con variables cuantificables definidas a priori. Los principales resultados arrojan que los docentes perciben que los problemas en el contexto educativo se explican por variables de disciplina y de convivencia y que dichos problemas varían según el lugar de procedencia de los estudiantes; así mismo, identifican diferencias en la forma de vinculación entre estudiantes nativos y extranjeros y que existe una relación significativa entre los estudiantes migrantes problemáticos y las dificultades para establecer relaciones de cooperación. Por otro lado, perciben bajo compromiso de apoderados de estudiantes migrantes. Se concluye que la percepción docente permite identificar los modos de constituir el pensamiento social y la construcción social de la realidad migratoria y que esto es relevante para predecir las barreras y, a su vez, los desafíos para configurar escuelas interculturales.
\end{abstract}

Palabras Clave: Educación; interculturalidad; percepción; migraciones; profesor

\section{Foreign students in the representation of teachers of a school in Santiago: elements to build an intercultural education.}

\begin{abstract}
The article addresses the perception of teachers around migrant students in a public school in Santiago, Chile. It is a quantitative, descriptive correlation study with a sample of 16 teachers. Data collection was carried out through an ad hoc instrument with quantifiable variables defined a priori. The main results show that teachers perceive that problems in the educational context are explained by variables of discipline and coexistence and that these problems vary according to the place of origin of the students; likewise, they identify differences in the form of linkage between native and foreign students and that there is a significant relationship between problematic migrant students and difficulties in establishing cooperative relationships. On the other hand, they perceive low commitment of guardians of migrant students. It is concluded that the teacher's perception allows to identify the ways of constituting the social thought and the social construction of the migratory reality and that this is relevant to predict the barriers and, at the same time, the challenges to configure intercultural schools.
\end{abstract}

Keywords: Education; interculturality; perception; migrations; teacher

\section{Estudantes estrangeiros na representação dos docentes numa escola de Santiago: elementos para uma educação intercultural}

Resumo: O artigo trata da percepção de ensino de estudantes migrantes numa escola pública de Santiago do Chile. 
Trata-se de um estudo quantitativo, descritivo-correlacional, com uma amostra composta por 16 professores. A coleta de dados foi realizada através de um instrumento ad hoc com variáveis quantificáveis definidas a priori. Os principais resultados mostram que os professores percebem que os problemas no contexto educacional são explicados por variáveis de disciplina e convivência e que esses problemas variam de acordo com o local de origem dos alunos; Da mesma forma, eles identificam diferenças na forma de vinculação entre estudantes nativos e estrangeiros e que existe uma relação significativa entre estudantes migrantes problemáticos e as dificuldades em estabelecer relações de cooperação. Por outro lado, eles percebem um escasso compromisso dos padres de estudantes migrantes. Conclui-se que a percepção do ensino permite identificar os modos de constituir o pensamento social e a construção social da realidade migratória e que isso é relevante para prever as barreiras e, por sua vez, os desafios para a configuração das escolas interculturais.

Palavras-chave: Educação; interculturalidade; percepção ;migrações; professor

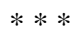

\section{Introducción}

Desde 1990 hasta hoy la migración latinoamericana a Chile se ha cuadruplicado. En efecto, entre 2005 y 2017 la migración incrementó en un 400\% (Mundaca, Fernández y Vicuña, 2018). De acuerdo con el informe del Observatorio iberoamericano sobre Movilidad Humana, Migraciones y Desarrollo (Rojas y Silva, 2016), este fenómeno se acentúo a partir del 2001, debido a procesos internacionales que posicionaron a Chile como uno de los principales países de destino de la migración en el contexto sudamericano: por un lado, el reforzamiento de la seguridad de las fronteras en el primer mundo por la amenaza terrorista del 11 de septiembre y, por otro, la profunda crisis económica y/o política que han atravesado algunos países de la región. Según el Censo 2017 (INE, 2018) los migrantes representan un 4,35\% del total de la población y el flujo migratorio está motivado principalmente por la estabilidad económica y política del país, siendo la región Metropolitana quien concentra el mayor porcentaje de la población migrante, con un 65.24\% (Departamento de extranjería y migraciones del Ministerio del interior y Seguridad pública, 2017). En este contexto, las características que definen el proceso migratorio que Chile ha venido experimentando durante la última década, ha puesto en evidencia el cambio estructural que ha emergido en la sociedad y la necesidad de repensar la forma de constituir escuelas, en tanto que en estas se manifiestan los efectos socio-culturales de cambio y convergen con una serie de dinámicas socioeducativas que son necesarias de atender para la promoción de contextos educativos eficaces y equitativos.

La cantidad de población migrante escolar ha aumentado. Según el Censo 2017, 788.939 migrantes se encuentran en el tramo de edad entre 0 y 14 años y 121.958 están entre los 15 y 24 años (Mundaca, Fernández y Vicuña, 2018). Dicha población requiere insertarse en contextos educativos y probablemente el mayor reto de las escuelas es resolver la inclusión de inmigrantes y hacer frente a todas las barreras (escasez de recursos, falta de formación en atención a la diversidad y convivencia escolar, discriminación, sobre-poblamiento de estudiantes, adecuaciones curriculares, entre otras) que ya existen en este campo. Sobre esto último, diversas investigaciones concluyen que precisamente en escuelas públicas del país es donde se manifiestan barreras relacionales y actitudinales en tanto los niños, niñas y jóvenes inmigrantes están expuestos a sufrir diversas experiencias de discriminación y racismo a nivel discursivo, «bromas» en base a sus características físicas y, agresiones verbales y/o físicas (Cortez, Loredo, Muñoz, Rodríguez y Vásquez, 2008; Pávez, 2012; Stefoni, Acosta, Gaymer y Casas-Cordero, 2008; Tijoux, 2013a) y que dicho racismo no sólo tiene raíz histórica sino que adquiere formas diversas dependiendo de los diferentes tiempos y contextos en los que se manifiesta. Así mismo, otra barrera tiene relación con elementos estructurales del sistema escolar debido a un currículum uniforme y la inexistencia de políticas de educación intercultural que den respuesta a la reconfiguración sociocultural de estos contextos (Mondaca, Muñoz, Gajardo y Gairín, 2018). En definitiva, la tarea y el desafío es la promoción de educación intercultural lo que se traduce en la adopción de un modelo que considera a la educación como responsable de dar respuestas a demandas sociales de equidad e inclusión social (Villalta, 2016).

En este ejercicio, los docentes son quienes lideran esta tarea en lo cotidiano y de manera directa, en tanto son actores claves en el aula. De ello, emana la importancia de poner en relieve las representaciones sociales de la persona docente y, específicamente, conocer cómo es su construcción de la realidad en torno a estudiantes migrantes y cómo esta configuración incide positiva o negativamente en las prácticas pedagógicas, en el clima educativo dentro y fuera del aula, en la convivencia escolar y en la relación estudiante-docente, entre otros aspectos. 
La importancia de estudiar la percepción de los docentes en torno a los estudiantes migrantes radica en que permite identificar y predecir los modos y procesos de constitución del pensamiento social, por medio del cual construyen la realidad social y su visión de mundo. Esto resulta vital en los procesos de «hacer» escuelas interculturales en donde el carácter de inclusión, el respeto y la valoración de la diversidad cultural estén presentes. Pero ¿qué pasa cuando las percepciones docentes son negativas o cargadas de juicios y sesgos?, ¿̇e puede promover la interculturalidad en estos contextos educativos? Bajo este escenario, es fundamental llevar a cabo un análisis crítico de la composición de las escuelas. Estos espacios son instituciones que se constituyen a través de las personas que las habitan y transmiten sus creencias, convicciones, percepciones y expectativas, entre otros elementos. Este estudio centra su interés precisamente en conocer la percepción docente acerca de estudiantes migrantes, así como la problematización de aquellos elementos y condiciones socioeducativas en estas materias tales como factores relacionales, actitudinales, y limitaciones estructurales de los sistemas escolares en la promoción de interculturalidad y, que pueden ser transversales a las escuelas públicas del país.

El marco institucional de educación en Chile a partir del 2012, con las nuevas bases curriculares, aborda el Curriculum de forma inclusiva, lo que conlleva que la o el docente debe considerar la diversidad presente en el aula al momento de llevar a cabo su trabajo pedagógico (Mineduc, 2018a). Esto implica pensar en las múltiples formas en que las y los estudiantes se aproximan al aprendizaje y al conocimiento, sin hacer discriminaciones por sus orígenes o características culturales, sociales, étnicas, religiosas, de género, de estilos de aprendizaje y de niveles de conocimiento (Mineduc, 2018a). Por tanto, una de las líneas de profundización es el rol de los docentes en la tarea de constituir escuelas interculturales.

\section{Fundamentos de educación intercultural}

Hablar de una educación intercultural implica distinguir multiculturalismo e interculturalidad, perspectivas teóricas que han intentado responder a la tendencia homogeneizadora del modelo educativo predominante durante el siglo XX y XXI, sustentado en una estructura de diferenciación y desigualdad social, a pesar de la diversidad cultural que lo conforma (Diez, 2004). El multiculturalismo, cuyo origen se ubica en los Estados Unidos, se entiende como un enfoque y/o política que interviene en el acompañamiento y desenvolvimiento de la dinámica social de las comunidades, por medio de relaciones simétricas e igualitarias, promoviendo la tolerancia y el respeto entre los diferentes grupos culturales (Diez, 2004; Cabral, 2009; Dietz, 2012). Sin embargo, este enfoque evidencia la persistencia de las desigualdades y la exclusión que afecta a grupos minoritarios, debido a que los colectivos culturales se mantienen aislados y «reproducen prácticas culturales de sus países de origen, sostienen relaciones con estos lugares y forman comunidades de connacionales en los países que residen» (Stefoni, C., Corvalán, J., Riedemann, A. y Stang, F, 2018, p.10).

En tanto, la interculturalidad, de mayor desarrollo en Europa y Latinoamérica, ha evolucionado respecto del discurso multiculturalista, convirtiéndose en una respuesta a contextos sociales y objetivos diversos, en particular para el caso de América Latina, (Stefoni, Stang y Riedemann, 2016). Por tanto, el concepto de interculturalidad surge como una respuesta a la condición multicultural de las sociedades latinoamericanas, vinculado a las demandas de los pueblos indígenas en contra de los estados nación. A su vez, es una propuesta política que va más allá de acompañar la dinámica social entre diferentes colectivos, promoviendo el diálogo en un marco de respeto entre estos grupos, para generar el encuentro y comunicación que no se daría naturalmente. En consecuencia, la interculturalidad, intenciona el encuentro entre diferentes culturas y saberes en condiciones de igualdad y que mediante el diálogo simétrico logran construir una sociedad que asume la diversidad (Diez, 2004, Walsh, 2002).

Es oportuno indicar que en el caso de América Latina, el enfoque intercultural se ha centrado en la formulación de programas de educación intercultural bilingüe, focalizado en las propias comunidades indígenas, sin haber logrado permear al sistema escolar en general. En efecto, la educación en contexto indígena se ha basado en prácticas monoculturales de manera transversal en todos los niveles del sistema educacional. La monoculturalidad se evidencia en el desconocimiento del contexto social y cultural de los estudiantes; así, la educación escolar pública insiste en la transmisión de prejuicios y actitudes racistas respecto a grupos indígenas y migrantes, consolidando la desvalorización hacia estos grupos . Por tanto, se debe pensar en la educación 
intercultural de manera crítica, entendida como un proyecto ético, político y epistémico, para la transformación de las bases que sostienen la asimetría y desigualdad en la sociedad y que se transmite a través de la escolarización (Quintriqueo, Quilaqueo, Lepe -Carrión, Riquelme, Gutiérrez, y Peña-Cortes, 2014; Arias-Ortega, Quintriqueo y Valdebenito, 2018).

En el caso de Chile, la educación intercultural ha estado vinculada, al igual que en el resto de Latinoamérica la educación de los pueblos indígenas, a través del programa «educación intercultural bilingüe EIB» propuesto durante la década de 1990 a partir de la promulgación de la Ley Indígena (Ley No 19.253), cuyo objetivo era preparar a los estudiantes indígenas para desenvolverse tanto en la sociedad de origen como en la sociedad globalizada (Ministerio de Planificación y Cooperación, Ley Nº19.253, 1993). Sin embargo, la falta de definición y conceptualización del programa, sumado a las asimetrías históricas con estos colectivos, han generado barreras en el diálogo entre culturas y ha afectado la calidad de los aprendizajes en los estudiantes indígenas (CIAE, 2011). Por lo tanto, la educación intercultural en el sistema escolar chileno debiese tener como propósito la construcción de un diálogo entre sociedades y culturas diferentes, más allá de los grupos indígenas, disminuir la monoculturalidad, superar el carácter colonial de la educación, implementación de prácticas pedagógicas fundadas en la consciencia crítica, así como la lucha contra el racismo y la discriminación (Sáez, 2006; Walsh, Viaña, y Tapia 2010).

De esta manera, la perspectiva intercultural en el espacio educativo no puede ceñirse al uso simplista del concepto, sino que debe ser transversal al sistema, siendo al mismo tiempo un proyecto político, cultural y educativo. En efecto, lo intercultural tiene una connotación positiva y mediatizada por la noción de escuelas nuevas, activas, abiertas y transformadoras y ello vincula macro procesos tales como adaptación, transformación y cambios institucionales; y a nivel micro, el intercambio, el mutuo enriquecimiento y la cooperación entre pares, entre otras cosas.

Bajo estos enfoques es que la educación intercultural se entenderá como:

«la reflexión sobre la educación, entendida como elaboración cultural, y basada en la valoración de la diversidad cultural. Promueve prácticas educativas dirigidas a todos y cada uno de los miembros de la sociedad en su conjunto. Propone un modelo de análisis crítico y de actuación que afecte a todas las dimensiones del proceso educativo. Se trata de lograr la igualdad de oportunidades (entendida como oportunidades de elección y de acceso a recursos sociales, económicos y educativos), la superación del racismo y la competencia intercultural en todas las personas, sea cual sea su grupo cultural de referencia» (Aguado Odina, 2004, p. 40)

Esta concepción de educación intercultural obedece a un enfoque desde el que se abordan las cuestiones relativas al tratamiento de la diversidad cultural en educación y que conlleva a la comprensión de que la diversidad se manifiesta más allá de los límites establecidos por razas, grupos étnicos o naciones y que además interacciona con una serie de variables socioeducativas tanto en contextos formales y otros menos estructurados. Por último, la promoción de escuelas interculturales obedece a procesos sociales y políticos de carácter dinámico que involucra a una serie de actores de la comunidad educativa, surtidos de identidades propias, creencias y representaciones sociales en torno a la diversidad sociocultural. Lo interesante es ponderar el nivel de contribución de estas representaciones sociales en la configuración de escuelas interculturales. Esta investigación se centra en la percepción de los docentes acerca de lo intercultural y de la presencia de sujetos migrantes en contextos educativos, y así identificar representaciones sociales sobre este fenómeno.

\section{Rol docente en la construcción de una comunidad escolar intercultural}

Previamente ha quedado en evidencia el alto flujo migratorio que Chile ha experimentado en las últimas décadas, no obstante esta investigación pone en relieve uno de los resultados de este cambio socio-cultural, el cual se relaciona con la presencia de niños, niñas y jóvenes inmigrantes de primera y segunda generación en escuelas chilenas, quienes traen consigo fuertes rasgos de la cultura familiar y de su país de procedencia, y que por tanto - bajo el supuesto de interculturalidad- es necesario un nuevo planteamiento acerca de la práctica 
educativa y la forma de construir contextos educativos que den respuesta a los cambios culturales que se han producido (Riedemann, Stefoni, 2015; Pávez, 2013; Tijoux, 2013a y b). Este nuevo contexto sociocultural reafirma la necesidad del modelo de educación intercultural, en donde las necesidades presentadas por los alumnos y alumnas no son tan solo pedagógicas, sino también de orden psicológico, político y social (Taylor, 1992).

En este escenario, es fundamental el papel de los docentes para conocer y dar significado a lo que es la interculturalidad y de qué manera se puede fomentar dentro y fuera del aula (Besalú, 2007). Dicho esto, el rol de los docentes en la educación intercultural se orienta al desarrollo de un paradigma en el que éstos sean críticos y conscientes de que su papel es de facilitadores, ejerciendo, siempre que sea necesario, la mediación, la argumentación y la resolución de conflictos (Gil, 2010). Así mismo, este rol debiese estar precedido por una formación pedagógica, es decir una investigación y análisis de estrategias didácticas que puedan impulsar y animar prácticas interculturales (Besalú, 2007). En la misma línea, una formación intercultural pensada para que el docente sepa reflexionar y posicionar un discurso neutral exento de discriminación y, a su vez, posicionar perspectivas que se distancien de la visión de cultura hegemónica («la chilena») por sobre otras. En este punto, las representaciones sociales de los docentes respecto a la inmigración y la diversidad cultural son esenciales para la formación intercultural, en tanto una actitud o un cambio de actitud- con base en las representaciones sociales- dirigida a desprenderse de prácticas naturalizadas que atenten contra la diversidad y su riqueza, permite crear nuevas maneras de situarse e intervenir en el día a día en las escuelas (Candau, 2008). Por tanto, más que hablar de estrategias interculturales, la práctica docente debiese estar centrada en el desarrollo de una conciencia que conlleve al desarrollo de competencias interculturales, y en efecto, transformar, la monoculturalidad de las prácticas pedagógicas (Vila, 2003, Arias-Ortega, Quintriqueo y Valdebenito, 2018). Dicho esto, el rol del docente se traduce en una serie de prácticas tales como: potenciar la comunicación entre personas de distintos orígenes, promoviendo espacio para este encuentro a partir de un trabajo heterogéneo y que sea cooperativo; eliminar los estereotipos y discriminaciones, fomentar la diversidad y los valores que nacen de la diversidad, respeto, tolerancia por su cultura y la del otro, desarrollar la comprensión de que el mundo es desigual y entender que se debe actuar de forma responsable en ello y plantear constantes cambios que conduzcan a la interculturalidad y al dinamismo de esta (Mineduc, 2017, 2018b; Gómez Lara, 2003).

\section{Representaciones sociales de los docentes frente a la inclusión de alumnos inmigrantes}

Teniendo claridad acerca de la necesidad de promover una educación intercultural a propósito de la diversidad cultural que caracteriza al Chile de hoy y del rol de los docentes en esta tarea, se torna necesario abordar las representaciones sociales de los docentes respecto a la inclusión de alumnos inmigrantes, en tanto que dichas representaciones predicen el desarrollo de algunos de los paradigmas de educación intercultural.

Dicho esto, el hecho de que la inmigración en Chile sea un fenómeno social de carácter reciente en cuanto a magnitud, explica el desconocimiento existente de estos colectivos y, específicamente, en el ámbito educativo surge la necesidad de repensar el sistema a propósito de la presencia de migrantes en estos espacios. Estos elementos (desconocimiento y rediseño de las escuelas) pueden dar origen a una percepción negativa por parte del profesorado y la comunidad escolar en general, en tanto se visualiza un problema- más que una oportunidad -para introducir cambios y, por ende, una tarea que se suma a la ya sobrecarga experimentada por el profesorado de escuelas públicas (Ortiz, 2008).

En esta misma línea, el acentuar la inclusión de inmigrantes como un problema puede explicarse a partir de la presencia de estereotipos, desconocimiento, falta de directrices para el desarrollo efectivo de escuelas inclusivas e interculturales. Ortiz (2008) sostiene que el incremento del alumnado extranjero en las aulas efectivamente está siendo percibido como una amenaza y visto como uno de los problemas principales del sistema educativo en tanto afecta la convivencia escolar y los procesos de enseñanza-aprendizaje. Dicho discurso, proveniente de los docentes, se sitúa desde una posición asimilacionista, según la cual las diferencias de cualquier orden deben ser suprimidas (Ortiz, 2008). Esta ideología puede inducir una actitud prejuiciosa, replicada por toda la comunidad educativa, que finalmente incide desfavorablemente en el contexto escolar y, específicamente, en las dinámicas que se gestan en el aula. Así mismo, bajo la lógica de suprimir diferencias y promover la igualdad entre alumnos, esta última puede ser mal entendida, en el sentido de intentar estandarizar los procesos educativos, descono- 
ciendo la multiculturalidad existente en las escuelas y, por ende, invisibilizando la riqueza que puede ofrecer la diversidad. Kaluf (2009) indica que gran parte de los docentes se encuentran poco preparados a propósito del carácter reciente del fenómeno migratorio en las aulas y tienden a considerar cualquier tipo de diferenciación como una forma de discriminación y, por tanto, trabajan bajo la ilusión del trato igualitario, desconociendo la diversidad en el aula y no utilizándola como una herramienta educativa. De igual modo, Castro (2012) señala que los docentes asumen que los niños y niñas migrantes no requieren de apoyo o tratos diferenciados, ya que son considerados como iguales a los estudiantes nativos y, por tanto, no modifican sus prácticas pedagógicas. Esto como consecuencia arroja la dificultad para desarrollar una cultura inclusiva al menos en dos elementos: construcción de una comunidad educativa intercultural y la presencia de valores inclusivos en las escuelas (Cerón, L. Pérez-Alvarado, M. y Poblete, R., 2017).

Por otro lado, cabe mencionar que las escuelas son un reflejo de la sociedad y a través de ellas también se puede observar una percepción sesgada y estereotipada hacia la población migrante, probablemente a causa del desconocimiento. En torno a esto, algunas investigaciones han concluido que se tiende a atribuir cualidades y/o defectos a alumnos migrantes según su lugar de procedencia (Segura, 2016). Estos hallazgos permiten esbozar la actitud pedagógica de los docentes hacia sus alumnos y, a su vez, pone en evidencia la dinámica que se establece en los espacios educativos. De esta manera, es probable observar una actitud más o menos negativa con alumnos proveniente de países de los cuales se tiene una percepción desfavorable a propósito de su cultura, sus tradiciones, sus características sociales o políticas. En esta línea, a partir de la misma investigación, se concluye que la percepción de docentes de la región de Rosario, en Argentina, acerca de alumnos peruanos, es favorable en comparación con alumnos de otras procedencias, en tanto se les adjudican cualidades tales como «ser más educados», «tranquilos», «aplicados», «inteligentes» y «agradecidos» (Segura, 2016). Asimismo, en otra investigación, se concluye que en el imaginario social de los habitantes de la región de Antofagasta, en Chile, existe una representación negativa de ciertos estudiantes extranjeros, como es el caso de los estudiantes colombianos, debido a la exposición de características negativas de personas asociadas al crimen, robo, tráfico de drogas y prostitución, en medios de comunicación masiva (Stang y Stefoni, 2016), situación que ha contribuido a la estigmatización de estos estudiantes.

Bajo este escenario, es posible predecir que la actitud pedagógica sea más favorable con aquellos alumnos de los que se posee una percepción positiva y una actitud pedagógica negativa con aquellos alumnos de los que se posee una percepción negativa.

En este contexto, puede ser frecuente la comparación entre alumnos inmigrantes a propósito del grado de desarrollo y/o subdesarrollo de los países que provienen y de cualquier otra característica asociada a la zona de origen y, por consiguiente, contrastando formas contrapuestas de vidas, de lo que se infieren, sesgadamente, características de personalidad comunes a todas las personas del mismo origen (Morgan, 1971). En correspondencia con lo anterior, en una investigación relacionada con las percepciones prejuiciosas de los docentes, se sostiene que los profesores ponen de manifiesto una forma de pensamiento esencialista que afirma que la cultura es algo que la gente «tiene» y que algunos «tienen mejor que otros», reproduciendo las relaciones de poder características de la ideología dominante y que perpetuán el estereotipo, la estigmatización y el racismo (Pettmann, 1992). Esta percepción esencialista de las diferencias y la visión estática de la cultura pueden desencadenar significativas barreras en la conformación de escuelas interculturales. Ante este panorama, nuevamente se pone el acento en el rol de los docentes y la comunidad escolar en totalidad- en la transformación de los sistemas educativos.

Por otra parte, es interesante señalar que la declaración de los docentes respecto a la inclusión de niños y niñas migrantes, es favorable y aceptada por una mayoría significativa; sin embargo, son las prácticas cotidianas las que develan las aprehensiones a propósito de los sesgos estereotipados, los imaginarios, las etiquetas esencialistas, el desconocimiento y la falta de elementos promotores en la constitución de una escuela inclusiva, entre otros aspectos (Cerón, L. Pérez-Alvarado, M. y Poblete, R., 2017). En este sentido, resulta fundamental abordar las percepciones docentes acerca de niños y migrantes, puesto que dichas concepciones ponen de manifiesto los elementos que son convenientes de transformar y cuáles son interesantes de perpetuar para dar respuesta a la necesidad de repensar las escuelas a propósito de la diversidad cultural que allí converge. 


\section{Metodología}

La investigación que aquí se presenta corresponde a un estudio de carácter cuantitativo que buscaba identificar la percepción docente respecto de la presencia de estudiantes migrantes en una escuela pública de la ciudad de Santiago.

El diseño de la investigación fue de carácter descriptivo-correlacional, en el que se analizaron dimensiones de la percepción docente tales como fortalezas y debilidades de estudiantes migrantes, barreras en el aula, rol de padres y/o apoderados, presencia de actitudes y/o conductas discriminatorias, relaciones sociales de estudiantes migrantes, dificultades de aprendizaje, aspectos culturales positivos y negativos, entre otros.

Para el levantamiento de información se utilizó un instrumento ad hoc construido para estos fines, de carácter anónimo, en formato escala tipo Likert y preguntas abiertas, las que posteriormente fueron codificadas y operacionalizadas.

La muestra estuvo conformada por 16 docentes (12 mujeres y 4 hombres). El muestreo fue por conveniencia (Otzen y Manterola , 2017) en tanto esta técnica permitió seleccionar aquellos casos accesibles y que aceptaron participar del estudio.

En cuanto al centro educacional donde se realizó el levantamiento de información, se indica que es un establecimiento municipal que cuenta con nivel de enseñanza de educación parvularia y enseñanza básica. Este centro educativo se ubica en la comuna de Santiago, característica importante debido a que esta es la segunda comuna de la región metropolitana con mayor población migrante, alcanzando un 27,55\% respecto del total de habitantes de esta (Mundaca, Fernández y Vicuña, 2018).

Los análisis estadísticos se realizaron a través del programa informático SPSS, específicamente análisis descriptivos y correlacionales.

Las consideraciones éticas para esta investigación contemplaron el anonimato de la escuela pública en donde se hizo la recolección de datos, así como también la confidencialidad en la identificación de los participantes. Estas decisiones tienen relación con evitar la estigmatización hacia estudiantes y docentes a propósito de la complejidad de los fenómenos migratorios que atraviesa nuestro país.

\section{Resultados}

Respecto a los análisis descriptivos, se pone en relieve que cerca del 43\% de los participantes concibe la educación intercultural como la «unión de varias culturas en favor de los aprendizajes» y, aproximadamente, el $21 \%$ la define como la «interacción de distintas culturas». Estos datos revelan que alrededor del $60 \%$ de la muestra tiene una concepción de la educación intercultural más cercana al concepto de multiculturalidad, el cual se utiliza para referirse especialmente a los inmigrantes que deben integrarse a un Estado nacional, y específicamente a una cultura predominante, «la chilena» cuya tradición cultural ha sido construida desde hace varios siglos (García, Pulido y Montes, 1997).

En palabras sencillas, la multiculturalidad se limita a los aspectos más curriculares, incluyendo diversos temas culturales en el Curriculum (tradiciones, costumbres, historia, entre otras cosas), dejando de lado la intervención en la sociedad. Por el contrario, la educación intercultural va un paso más allá, en tanto entiende la importancia del carácter social de las propuestas educativas con el fin de establecer un análisis crítico de aquellos elementos que propician una reconfiguración sociocultural la cual no se traduce en una mera actividad multicultural, así como la reflexión que promueva el cambio de los alumnos y alumnas logrando la adquisición de un punto de vista más relativo sobre el mundo, el establecimiento de relaciones simétricas e inclusivas. En relación con la comprensión de la educación intercultural, solo el 21\% de los participantes la concibe de esta manera. 
Este dato es importante para comprender la concepción de los docentes y cómo esta promueve o frena la configuración de escuelas públicas interculturales, a propósito del cambio sociocultural que se ha experimentado en los últimos años en los contextos educativos del país. Dicho eso, se torna fundamental el cambio paradigmático de docentes respecto a la importancia de comprender la educación intercultural para así adoptar estrategias socioeducativas que promuevan la inclusión.

Así mismo, el concepto de multiculturalidad, en el establecimiento donde se recolectaron datos, queda en evidencia debido a las actividades declaradas por los docentes para la promoción del intercambio cultural. El 35,7\% de los docentes señala que se llevan a cabo actividades que articulan intercambio de experiencias con asignaturas, el 28,6\% declara que el intercambio cultural se genera a través de la implementación de festivales, exposiciones y ferias costumbristas; mientras que solo un 14, 3\% percibe la importancia de potenciar la convivencia escolar y la cooperación.

\section{a) Percepción docente hacia el comportamiento de estudiantes inmigrantes en el contexto educativo.}

En cuanto a la percepción de los docentes respecto al comportamiento de los estudiantes migrantes, el $57,2 \%$ de los docentes declara que los mayores problemas en el contexto educativo se deben a temas de disciplina, comportamiento y el no respeto de las normas de convivencia y el 50\% indica que dichos problemas se generan con estudiantes de origen colombiano, el 14,3\% con estudiantes venezolanos, el 7,1\% con población peruana y el 7,1\% con población de estudiantes haitianos y dominicanos.

Se realizó una correlación entre las variables «mayores problemas en el aula con estudiantes según su procedencia» y «las características culturales generan dificultades en las relaciones sociales» y se alcanzó un r .704 con un nivel de significancia del 0.007. Dicha tendencia pone de manifiesto la atribución de conflictos a una condición de origen cultural/territorial, es decir, la población docente atribuye los problemas comportamentales a una condición esencialista vinculada a las características socio-culturales de los estudiantes migrantes (comportamientos, actitudes y estilos vinculares que se adquieren en el seno cultural), lo que puede desencadenar un estereotipo hacia poblaciones determinadas y, por tanto, una actitud negativa que atente contra la convivencia escolar, el espacio del aula y la relación docente-estudiantes. La tabla 1 muestra el análisis correlacional.

Tabla 1.

Correlación entre problemas con estudiantes según su país de procedencia y características culturales.

\begin{tabular}{|c|c|c|c|}
\hline \multicolumn{4}{|c|}{ Correlaciones } \\
\hline & & $\begin{array}{l}\text { Mayores problemas en } \\
\text { el contexto educativo } \\
\text { con estudiantes según } \\
\text { su procedencia }\end{array}$ & $\begin{array}{l}\text { Las características } \\
\text { culturales generan } \\
\text { dificultades en las } \\
\text { relaciones sociales. }\end{array}$ \\
\hline \multirow{3}{*}{$\begin{array}{l}\text { Mayores problemas en el } \\
\text { aula con estudiantes según } \\
\text { su procedencia }\end{array}$} & $\begin{array}{l}\text { Correlación de } \\
\text { Pearson }\end{array}$ & 1 &, $704^{* *}$ \\
\hline & Sig. (bilateral) & &, 007 \\
\hline & $\mathrm{N}$ & 15 & 16 \\
\hline \multirow{3}{*}{$\begin{array}{lr}\text { Las } & \text { características } \\
\text { culturales } & \text { generan } \\
\text { dificultades en las } \\
\text { relaciones sociales. }\end{array}$} & $\begin{array}{l}\text { Correlación de } \\
\text { Pearson }\end{array}$ &, $704^{* *}$ & 1 \\
\hline & Sig. (bilateral) &, 007 & \\
\hline & $\mathrm{N}$ & 15 & 16 \\
\hline
\end{tabular}

\section{b) Percepción docente hacia la relación entre estudiantes inmigrantes y nativos}

Probablemente esta dimensión de análisis es la que permite obtener mayores resultados significativos y comprensivos respecto de la percepción docente. 
En cuanto a las relaciones sociales entre estudiantes nativos (chilenos) y estudiantes migrantes (latinoamericanos), los docentes perciben diferencias en las formas de vinculación y esta diferencia se relaciona con la presencia de actitudes y/o conductas discriminatorias. Se realizó un análisis correlacional entre las variables «actitud y/o conductas discriminatorias» y «diferencia en las formas de relacionarse entre nativos e inmigrantes» y se obtuvo un r .658 con un nivel de significancia de 0.011 lo que permite concluir que a mayor diferenciación en las formas de relacionarse entre nativo e inmigrantes mayor es la presencia procesos discriminatorios. Esto podría estar desencadenado por las dificultades en la convivencia escolar y por una ineficaz integración de la comunidad educativa. La tabla 2 muestra el análisis correlacional.

Tabla 2.

Correlación entre actitud y/o conductas discriminatorias y diferencias en la forma de relacionarse de nativos e inmigrantes.

\begin{tabular}{|c|c|c|c|}
\hline \multicolumn{4}{|c|}{ Correlaciones } \\
\hline & & $\begin{array}{l}\text { Actitud } \quad y / o \\
\text { conductas } \\
\text { discriminatorias }\end{array}$ & $\begin{array}{l}\text { ¿existen diferencias en la } \\
\text { forma de relacionarse de } \\
\text { nativos e inmigrantes? }\end{array}$ \\
\hline \multirow{3}{*}{$\begin{array}{l}\text { Actitud } \quad y / o \\
\text { conductas } \\
\text { discriminatorias }\end{array}$} & $\begin{array}{l}\text { Correlación de } \\
\text { Pearson }\end{array}$ & 1 & $658^{*}$ \\
\hline & Sig. (bilateral) & &, 011 \\
\hline & $\mathrm{N}$ & 16 & 16 \\
\hline \multirow{3}{*}{$\begin{array}{lr}\text { ¿existen } & \\
\text { diferencias en } & \text { la } \\
\text { forma } & \text { de } \\
\text { relacionarse } & \text { de } \\
\text { nativos } & \text { e } \\
\text { inmigrantes? } & \end{array}$} & $\begin{array}{l}\text { Correlación de } \\
\text { Pearson }\end{array}$ &, $658^{*}$ & 1 \\
\hline & Sig. (bilateral) &, 011 & \\
\hline & $\mathrm{N}$ & 16 & 16 \\
\hline
\end{tabular}

Elaboración propia

Así mismo, otro análisis permitió establecer que las formas de relacionarse que establecen los estudiantes migrantes afecta la convivencia escolar y que dicha convivencia escolar podría estar mediatizada por la presencia de actitudes y/o conductas discriminatorias. La correlación entre ambas variables arrojó un r -,619 con un nivel de significancia de 0.018. La tabla 3 muestra la correlación obtenida.

Tabla 3.

Correlación entre actitud y/o conductas discriminatorias y las relaciones de los inmigrantes afecta la convivencia escolar.

\begin{tabular}{|c|c|c|c|}
\hline \multicolumn{4}{|c|}{ Correlaciones } \\
\hline & & $\begin{array}{l}\text { Actitud } \quad y / o \\
\text { conductas } \\
\text { discriminatorias }\end{array}$ & $\begin{array}{l}\text { la forma de } \\
\text { relacionarse } \\
\text { de los } \\
\text { inmigrantes } \\
\text { afecta la } \\
\text { convivencia } \\
\text { escolar }\end{array}$ \\
\hline \multirow{3}{*}{$\begin{array}{l}\text { Actitud } y / o \text { conductas } \\
\text { discriminatorias }\end{array}$} & Correlación de Pearson & 1 &,$- 619^{*}$ \\
\hline & Sig. (bilateral) & &, 018 \\
\hline & $\mathrm{N}$ & 16 & 16 \\
\hline \multirow{3}{*}{$\begin{array}{l}\text { la forma de relacionarse } \\
\text { de los inmigrantes afecta } \\
\text { la convivencia escolar }\end{array}$} & Correlación de Pearson &,$- 619^{*}$ & 1 \\
\hline & Sig. (bilateral) &, 018 & \\
\hline & $\mathrm{N}$ & 16 & 16 \\
\hline
\end{tabular}


Un análisis significativo respecto a la percepción docente sobre la relación entre nativos y migrantes, es la diferencia por sexo. Se hizo una correlación entre las variables «sexo de los docentes» y «formas de relacionarse de los inmigrantes afecta la convivencia escolar». Los resultados del análisis arrojaron un r .609 con un nivel de significancia del 0.021 , lo que indica que existe una percepción diferenciada entre docentes de sexo femenino y docentes de sexo masculino. No obstante, es importante precisar que la distribución de la muestra por genero no es homogénea existiendo más mujeres que hombres por lo que esta limitante debe ser considerada en el análisis de resultados.

Por otro lado, al analizar las variables «estudiantes migrantes problemáticos» e «inmigrantes tienen dificultades para establecer relaciones de cooperación con otros estudiantes», se obtuvo un $\mathrm{r}$-.762 con una significancia del 0.002, lo que se traduce en una percepción docente negativa hacia los inmigrantes y probablemente atribuible a su condición de migrantes (condición esencialista) y esto, a su vez, dificulta establecer relaciones sociales enmarcadas en el compañerismo entre estudiantes. Esta conclusión se re-afirma con otro análisis correlacional entre la variable «inmigrantes tienen dificultades para establecer relaciones de cooperación con otros estudiantes» y «aspectos culturales negativos». La correlación entre ambas variables arrojó un r .613 con una significancia de 0.020 lo que indica la atribución esencialista y negativa hacia la población migrante por la condición misma de ser extranjero.

Las tablas 4 y 5 muestran las respectivas correlaciones.

Tabla 4.

\section{Correlaciones entre «estudiantes migrantes problemáticos» e «inmigrantes tienen dificultades para establecer relaciones de cooperación con otros estudiantes»}

\begin{tabular}{|c|c|c|c|}
\hline \multicolumn{4}{|c|}{ Correlaciones } \\
\hline & & $\begin{array}{l}\text { Inmigrantes } \\
\text { tienen } \\
\text { dificultades } \\
\text { para } \\
\text { establecer } \\
\text { relaciones de } \\
\text { cooperación } \\
\text { con otros } \\
\text { estudiantes }\end{array}$ & $\begin{array}{l}\text { Los } \\
\text { estudiantes } \\
\text { inmigrantes } \\
\text { son } \\
\text { problemáticos }\end{array}$ \\
\hline \multirow{3}{*}{$\begin{array}{l}\text { Inmigrantes tienen } \\
\text { dificultades } \\
\text { establecer relaciones de } \\
\text { cooperación con otros } \\
\text { estudiantes }\end{array}$} & Correlación de Pearson & 1 &,$- 762^{* *}$ \\
\hline & Sig. (bilateral) & &, 002 \\
\hline & $\mathrm{N}$ & 16 & 16 \\
\hline \multirow{3}{*}{$\begin{array}{lr}\text { Los } & \text { estudiantes } \\
\text { inmigrantes } & \text { son } \\
\text { problemáticos } & \end{array}$} & Correlación de Pearson &,$- 762^{* *}$ & 1 \\
\hline & Sig. (bilateral) &, 002 & \\
\hline & $\mathrm{N}$ & 16 & 16 \\
\hline
\end{tabular}

Elaboración propia 
Tabla 5.

Correlaciones entre «inmigrantes tienen dificultades para establecer relaciones de cooperación con otros estudiantes» $y$ «aspectos culturales negativos»

\begin{tabular}{|c|c|c|c|}
\hline \multicolumn{4}{|l|}{ Correlaciones } \\
\hline & & $\begin{array}{l}\text { Aspectos } \\
\text { culturales } \\
\text { negativos }\end{array}$ & $\begin{array}{l}\text { Inmigrantes } \\
\text { tienen } \\
\text { dificultades } \\
\text { para } \\
\text { establecer } \\
\text { relaciones de } \\
\text { cooperación } \\
\text { con otros } \\
\text { estudiantes }\end{array}$ \\
\hline \multirow{3}{*}{$\begin{array}{l}\text { Aspectos } \\
\text { negativos }\end{array}$} & Correlación de Pearson & 1 &, $613^{*}$ \\
\hline & Sig. (bilateral) & &, 020 \\
\hline & $\mathrm{N}$ & 16 & 16 \\
\hline \multirow{3}{*}{$\begin{array}{l}\text { Inmigrantes tienen } \\
\text { dificultades } \\
\text { establecer relaciones de } \\
\text { cooperación con otros } \\
\text { estudiantes }\end{array}$} & Correlación de Pearson &, $613^{*}$ & 1 \\
\hline & Sig. (bilateral) &, 020 & \\
\hline & $\mathrm{N}$ & 16 & 16 \\
\hline
\end{tabular}

Elaboración propia

\section{c) Percepción docente hacia el proceso académico de estudiantes inmigrantes en aula.}

En cuanto a la percepción docente acerca de los problemas que se dan en el aula con estudiantes migrantes, se indica que el 21,4\% declara que las formas de expresión y comunicación es uno de los principales ejes problemáticos, mientras que el 14,3\% percibe que los problemas con estudiantes inmigrantes se explican por su condición misma de migrantes. A partir de esto -nuevamente- queda en evidencia que las representaciones sociales de los docentes son mediatizadas por elementos esencialistas y esto permite dilucidar la eventual presencia de prejuicios y estigmatización hacia estos colectivos.

Respecto a la especificidad de los problemas que se manifiestan en el aula, el 64,3\% de los docentes refiere que estos se desencadenan en el segundo ciclo de educación básica, es decir, entre estudiantes de 11 y 13 años de edad. Así mismo, el 21,4\% de los docentes indica que los problemas en el aula se explican por la dificultad en la adaptación al sistema escolar; el 21,4\% por déficit en el aprendizaje; el 21,4\% por falta de recursos pedagógicos; el 14,3\% por diferencias culturales y otro 14,3 por lenguaje y comunicación.

Respecto a las dificultades en el aula asociadas al déficit en el aprendizaje, se realizó un análisis correlacional entre variables «mayores problemas en el aula con estudiante según su procedencia» y «estudiantes inmigrantes presentan mayores dificultades de aprendizaje que los chilenos», lo que arrojó un r .537 con una significancia de 0.059. Este análisis permite concluir que los docentes perciben que los estudiantes inmigrantes tienen déficit de los aprendizajes, asociado a su país de procedencia -y probablemente la percepción es que dicho déficit es mayor en la población inmigrante que en la población de estudiantes nativos. La tabla 6 muestra el análisis correlacional. 
Tabla 6.

\section{Correlación entre «mayores problemas en el aula con estudiante según su procedencia» y «estudiantes inmigrantes presentan mayores dificultades de aprendizaje que los chilenos»}

\begin{tabular}{|c|c|c|c|}
\hline \multicolumn{4}{|c|}{ Correlaciones } \\
\hline & & $\begin{array}{l}\text { Mayores } \\
\text { problemas en el } \\
\text { aula con } \\
\text { estudiantes } \\
\text { según su } \\
\text { procedencia }\end{array}$ & $\begin{array}{l}\text { Las } \\
\text { características } \\
\text { culturales } \\
\text { generan } \\
\text { dificultades en } \\
\text { las relaciones } \\
\text { sociales. }\end{array}$ \\
\hline \multirow{3}{*}{$\begin{array}{l}\text { Mayores problemas en el } \\
\text { aula con estudiantes según } \\
\text { su procedencia }\end{array}$} & Correlación de Pearson & 1 &, $704^{* *}$ \\
\hline & Sig. (bilateral) & &, 007 \\
\hline & $\mathrm{N}$ & 16 & 16 \\
\hline \multirow{3}{*}{$\begin{array}{lr}\text { Las } & \text { características } \\
\text { culturales } & \text { generan } \\
\text { dificultades } & \text { en las } \\
\text { relaciones sociales. }\end{array}$} & Correlación de Pearson &, $704^{* *}$ & 1 \\
\hline & Sig. (bilateral) &, 007 & \\
\hline & $\mathrm{N}$ & 16 & 16 \\
\hline
\end{tabular}

Elaboración propia

\section{d) Percepción docente hacia el rol de apoderados}

Otro elemento de análisis es la percepción docente hacia la participación de padres y/o apoderados en el proceso educativo de sus estudiantes, en tanto se entiende la importancia de los adultos responsables en el proceso de enseñanza-aprendizaje de niños y niñas. En cuanto a esta apreciación, el 64,3\% de los docentes refiere un bajo compromiso por parte de apoderados y dentro de las explicaciones que ellos aluden a dicha falta de compromiso, el 50\% señala que hay una baja participación y presencia de apoderados en la comunidad escolar, un 21,4\% refiere el exceso de trabajo y el 7,1\% revela que los apoderados no acatan las normas de la escuela. Así mismo, el 71,4\% de los docentes relativiza las habilidades de los padres para ejercer el rol de apoderados/as, en tanto indican indecisión al momento de clasificarlos como poseedores de altas o bajas habilidades.

Al momento de establecer análisis correlacionales no se obtuvo ningún dato relevante.

\section{e) Enfoque de educación intercultural}

Finalmente, respecto a si el establecimiento educativo posee un modelo de educación intercultural, el $42.8 \%$ de los docentes señala que no poseen un modelo intercultural, un 35,7\% no tiene claridad sobre si existe o no educación intercultural en el centro educativo y un $21.4 \%$ aprecia que sí poseen un modelo de educación intercultural. Sin embargo, en este último punto es importante recodar que más del 40\% de los docentes concibe la educación intercultural no bajo la premisa propia de la interculturalidad, sino más bien desde la multiculturalidad, por lo que podría ser que el porcentaje que cree que sí operan bajo un enfoque intercultural, en verdad lo estén haciendo bajo el enfoque de multiculturalidad.

En esta misma línea de la educación intercultural se constató una relación estadística entre las variables «La escuela no posee un modelo de educación intercultural» y «estudiantes migrantes no son dóciles». Se realizó una correlación con ambas variables y se obtuvo un r .727 con un nivel de significancia del 0.003 lo que indica que la percepción podría estar representada por la creencia de que los estudiantes migrantes no son obsecuentes y que dicha actitud está mediatizada o no se puede abordar en tanto no existe un protocolo o un enfoque de educación intercultural que reconozca las diferencias y promueva la inclusión. La tabla 7 muestra la correlación. 
Tabla 7.

Correlación entre «la escuela no posee un modelo de educación intercultural» y «estudiantes migrantes no son dóciles»

\begin{tabular}{|l|l|l|l|}
\hline \multicolumn{2}{|c|}{ Correlaciones } \\
\hline \\
\end{tabular}

\section{Discusión y Conclusiones}

Como punto de partida se declara lo fundamental del enfoque de interculturalidad y su aplicación al contexto educativo, en tanto va más allá de las nociones de tolerancia y convivencia armónica, pues pone en discusión las relaciones de asimetría y subalternización existentes en el orden social vigente y, bajo este escenario, promueve la inclusión, el respeto de los derechos fundamentales de toda la comunidad educativa y favorece la toma de acciones respecto a aquellas barreras de exclusión que afectan a estudiantes por su condición de migrantes, por lo tanto, no es suficiente con garantizar el acceso a la escuela, sino que también la participación efectiva- en todos los niveles- de la comunidad. Dicho esto, lo relevante de esta investigación en torno a la percepción docente sobre el enfoque intercultural es la divergencia que se aprecia sobre el paradigma que debiese definir la estructura de una escuela intercultural. En este sentido, la percepción docente se acerca a lo que se denomina multiculturalidad y esta tiene mayor relación con la legitimación e interacción de diversas culturas en un espacio educativo, no obstante, no abandona la homogeneización de los procesos de enseñanzaaprendizaje y/o las lógicas multidimensionales de la convivencia escolar. Y más aún, invisibiliza la necesidad de dar un salto cualitativo respecto a las categorizaciones de estudiantes en función de etiquetas esencialistas, sesgos culturales, la no profundización en el conocimiento de otras culturas y el respeto por la diferenciación y la diversidad.

Esta conclusión cobra aún más valor a partir de los resultados obtenidos en esta investigación, la cual queda de manifiesto en a lo menos dos puntos. El primero es que las acciones que lleva a cabo la escuela para visibilizar la diversidad cultural se reducen a espacios culinarios y ferias costumbristas, entre otros, lo que puede desembocar en la folclorización de las culturas e invisibilizando el uso como herramientas para la transformación curricular y valórica que promueva la superación de visiones restringidas. Y el segundo punto se refiere a que los y las docentes generan representaciones sociales en torno a los y las estudiantes migrantes mediatizadas por etiquetas esencialistas y sesgos que podrían atentar en la promoción de la constitución de una escuela inclusiva bajo el enfoque intercultural. Estas etiquetas esencialistas tienden a atribuir a los migrantes una serie de déficit académicos y de comportamiento que pueden dificultar el proceso de inclusión y su plena participación en el contexto educativo. Si bien es cierto que gran parte de los docentes visualiza la importancia del respeto y la inclusión de los diferentes colectivos, es en la práctica cotidiana donde queda en evidencia la normalización de los sesgos, las representaciones sociales y los juicios a estudiantes dados por su origen o condición de migrantes. Y, por cierto, dichas representaciones son parte de la configuración que la sociedad ha ido construyendo de manera sistemática hacia determinados colectivos (Stang y Stefoni, 2016). Tal es el caso de la percepción de los docentes acerca de los estudiantes colombianos, a quienes aprecian como mayormente conflictivos en comparación a otros estudiantes de diferente origen o asumen que son las características culturales las que desencadenan problemas en las relaciones sociales entre nativos y migrantes o que los estudiantes migrantes presentan mayores problemas de aprendizaje que la población chilena. 
Este panorama debiese llevar necesariamente a la reflexión respecto del cambio en la mirada comprensiva de los procesos interculturales. El problema no es que las diferencias culturales generen barreras per se, sino que el dilema radica en la dificultad para relacionarse desde la diversidad o abandonar la estigmatización del lenguaje y de categorizarlo como un problema y reemplazarlo por la aceptación de las diferencias en el lenguaje, precisamente a propósito de la diversidad.

Por otro lado, el atribuir una concepción negativa al comportamiento de los estudiantes a propósito de características culturales, asienta la idea de que el imaginario de los docentes está marcado por estereotipos y estos se configuran como barreras para comprender la diversidad de estudiantes y sus formas de vincularse. Bajo este escenario, probablemente, el gran problema no es la diversidad de comportamientos, sino la falta de recursos pedagógicos para mejorar la convivencia escolar y abandonar la perspectiva normativa y excluyente que deriva de una concepción uniforme de los procesos educativos y comportamentales. En este punto se destaca que un porcentaje de docentes, aunque no mayoritaria, problematiza la falta de recursos pedagógicos y posiciona las dificultades en la estructura del contexto educativo.

Pese a que esta investigación no indaga en los efectos de las representaciones sociales en el espacio educativo y su incidencia en el proceso enseñanza-aprendizaje, sí se pueden predecir o al menos identificar barreras en torno a la inclusión, al reconocimiento de la diversidad y a la dificultad para implementar estrategias pedagógicas no homogeneizadas. En este sentido, la investigación no concluye que los docentes acentúen el carácter negativo de la inclusión de la diversidad y específicamente la inclusión de migrantes, pero sí se puede asentar la percepción negativa de algunos elementos que podrían afectar el desarrollo de una educación con enfoque de derechos humanos done se reconozca al extranjero como sujeto de derechos, la ausencia de valores en la línea de inclusión, la relación docente-estudiantes y la convivencia escolar; además del rendimiento académico y el éxito pedagógico.

A la luz de las conclusiones de esta investigación se visualizan retos necesarios de asumir para la promoción efectiva de escuelas interculturales. Aunque queda mucho por avanzar, se reconoce el esfuerzo sistemático de promover el cambio paradigmático en la forma de estructurar escuelas a propósito del cambio sociocultural que ha venido experimentado el país y la evidente diversidad cultural que seguirá creciendo exponencialmente. Si bien es cierto que el marco es restringido en materia de recursos de toda índole y políticas de educación incipientes en estas materias; lo estructural probablemente tiene relación con atender a la idiosincrasia de las escuelas y a la diversidad que en cada una ellas convergen. En este escenario uno de los tantos elementos a considerar es el rol de los docentes, actores claves de la cotidianidad de los espacios educativos. En la medida en que las representaciones sociales estén construidas sobre la base de sesgos culturales, lógicas esencialistas, bajo conocimiento, agotamiento y condiciones poco reconfortables para el quehacer docente, se perpetuará una percepción estereotipada hacia colectivos diversos culturalmente. Abandonar los sesgos culturales sin duda conlleva un proceso complejo y en dicho ejercicio se hace difuso establecer el límite entre lo correcto y aquello que no lo es; sin embargo, resulta atingente promover el respeto de los docentes por su propia labor pedagógica como eje central para un clima adecuado para el aprendizaje.

Dicho eso, el fortalecer los procesos de formación docente, en formación inicial o en ejercicio, respecto al enfoque intercultural es una estrategia que contribuye al contexto de las escuelas públicas del país. El enfoque intercultural conlleva a posicionar la importancia de la diversidad y la inclusión no solo para mejorar la convivencia escolar sino también como herramientas pedagógicas en el aula. Y bajo esta premisa, también es imperiosa la necesidad de avanzar en algunas reflexiones respecto del rol del docente en el nuevo concepto de escuelas y los desafíos en el campo de acción en dichos contextos.

Para ello es vital el pronunciamiento de los organismos públicos y las estructuras políticas en sus diferentes niveles (ministerial, municipal y local) para la generación de redes de apoyo a las escuelas y el respaldo claro a un paradigma de escuelas interculturales. En este sentido es fundamental el rol del Estado y el compromiso y la responsabilidad de todos los actores involucrados, en particular de los formadores de los actuales y futuros docentes. 


\section{Bibliografía}

Aguado Odina, T. (2004). Investigación en educación intercultural. Educatio, 22, 39-57. Recuperado de: https:// revistas.um.es/educatio/article/view/98

Arias-Ortega, K., Quintriqueo, S. \& Valdebenito, V. (2018). Monoculturalidad en las prácticas pedagógicas en la formación inicial docente en La Araucanía, Chile. Educação e Pesquisa, (44), 1-19.doi: 10.1590/s16784634201711164545

Besalú, X. (2007). Educar en Sociedades pluriculturales. Barcelona, España: Wolters Kluwer.

Cabral, E. D. (2009). Multiculturalismo y Educación. Cultura y representaciones sociales, 4(7), 27-54. Recuperado de: http://www.revistas.unam.mx/index.php/crs/article/view/16402/15611

Candau, V. (2008). Multiculturalismo e educação: desafio para prática pedagogica. Pétropolis, Brasil: Rj Vozes.

Castro, C. (2012). Inmigración, hoy: El reflejo de las representaciones sociales de los/las docentes en la cultura, política y las prácticas de la escuela en el marco de la diversidad cultural. Santiago, Chile: Universidad de Central.

Cerón, L. Pérez-Alvarado, M., Poblete, R. (2017). Percepciones docentes en torno a la Presencia de niños y niñas migrantes en Escuelas de Santiago: retos y desafíos para la inclusión. Revista Latinoamericana de Educación Inclusiva, 11(2), 233-246.doi: 10.4067/S0718-73782017000200015

CIAE. PEIB-ORÍGENES. (2011). Estudio sobre la implementación de la Educación Intercultural Bilingüe. Santiago, Chile: Ministerio de Educación.

Cortez, A., Loredo, P., Muñoz, C., Rodríguez, M. L., \& Vásquez, M. E. (Eds.) (2004). Niños y Niñas Inmigrantes en Chile: Derechos y realidades. Fundación Anide- Colectivo Sin Fronteras: Santiago.

Departamento de Extranjería y Migración del Ministerio del Interior y Seguridad Pública (2017). Reporte Migratorio. Santiago, Chile: DEM.

Diez, M. (2004). Reflexiones en torno a la interculturalidad. Cuadernos de Antropología Social, (19),191-213. Recuperado de: https://www.redalyc.org/pdf/1809/180913911012.pdf

Dietz, G. (2012). Multiculturalismo, interculturalidad y diversidad en educación. Una aproximación antropológica. Ciudad de México, México:Fondo de Cultura Económica.

García, J., Pulido, P., Montes, A. (1997). La educación multicultural y el concepto de cultura. Revista iberoamericana de educación, (13), 223-256. Recuperado de: http://red.pucp.edu.pe/ridei/wp-content/uploads/biblioteca/100108.pdf

Gil, I. (2010). Cultural diversity in compulsory education: An overview of the context of Madrid. Intercultural Education, 21(4), 299-315. doi: 10.1080/14675986.2010.506022

Gómez Lara, J. (2003). Los productos humanos, instrumentos de cambio para la educación. Recuperado de http:/ /www.aulaintercultural.org/conocer/pdfs/productos.pdf

Instituto Nacional de Estadísticas (INE) (2015). Anuario de estadísticas vitales de 2015. Santiago. Recuperado de https://www.ine.cl/docs/default-source/publicaciones/2017/s\%C3\%ADntesis-de-estad\%C3\%ADsticas-vitales-2015.pdf?sfvrsn=7.

Kaluf, M. (2009). Niños inmigrantes peruanos en la escuela chilena. Santiago, Chile: Universidad de Chile. 
Mc Laren, P. (2001). Multiculturalismo crítico. Sao Paulo, Brasil: Cortez.

Ministerio de Educación de Chile (MINEDUC). (2018a). Educación Intercultural. Santiago. Recuperado de http:/ /peib.mineduc.cl/institucionalidad/

Ministerio de Educación de Chile. (2018b). Mapa del estudiantado extranjero en el sistema escolar chileno (20152017). Santiago. Recuperado de https://www.mineduc.cl/wpcontent/uploads/sites/19/2018/05/ MAPA_ESTUDIANTES_EXTRANJEROS_SISTEMA_ESCOLAR_CHILENO_2015_2017.pdf

Ministerio de Educación. (2017). Orientaciones técnicas para la inclusión educativa de estudiantes extranjeros. Recuperado de https://www.mineduc.cl/wpcontent/uploads/sites/19/2017/12/Orientaciones-estudiantes-extranjeros-21-12-17.pdf

Mondaca, C., Muñoz, W., Gajardo, Y. y Gairín, J. (2018). Estrategias y prácticas de inclusión de estudiantes migrantes en las escuelas de Arica y Parinacota, frontera norte de Chile. Revista Estudio Atacameños Arqueología y Antropología Surandinas, (57), 181-201. doi: 10.4067/S0718-10432018005000101.

Morgan, L. (1971). La sociedad primitiva. Madrid, España: Ayuso.

Mundaca, P., Fernández, N., y Vicuña, J. (2018). Migración en Chile un análisis desde el censo 2017. Santiago, Chile: Servicio Jesuita a Migrantes.

Ortiz, M. (2008). Inmigración en las aulas: percepciones prejuiciosas de los docentes. Papers, (87), 253-268. doi: 10.5565/rev/papers/v87n0.805

Otzen, T.,Manterola, C. (2017). Técnicas de Muestreo sobre una población a estudio. International Journal of Morphology, 35(1), 227-232. doi: 10.4067/S0717-95022017000100037

Pávez, I. (2012). Inmigración y racismo: experiencias de la niñez peruana en Santiago de Chile. Si Somos Americanos, (12), 75-99. doi: 10.4067/S0719-09482012000100004

Pávez, I. (2013). Los significados de «ser niña y niño migrante»: conceptualizaciones desde la infancia peruana en Chile. Polis, 12 (35), 183-210. doi:10.4067/S0718-65682013000200009

Pettmann, J. (1992). Living in the margins: racism, sexism and feminism in Australia. Sydney, N.S.W: Allen \& Unwin.

Quintriqueo, S., Quilaqueo, D., Lepe -Carrión, P., Riquelme, E., Gutiérrez, M. \& Peña-Cortes, F. (2014). Formación del profesorado en educación intercultural en América Latina. El caso de Chile. Revista Electrónica Interuniversitaria de Formación del Profesorado, 17(2), 201-217. doi: 10.6018/reifop.17.2.198021

Riedemann, A., Stefoni, C. (2015). Sobre el racismo, su negación, y las consecuencias para una educación anti-racista en la enseñanza secundaria chilena. Polis, Revista Latinoamericana (42), 191-216. doi: 10.4067/S071865682015000300010

Rojas, N., Silva, C. (2016). La migración en Chile: breve reporte y caracterización. Madrid. Observatorio Iberoamericano sobre Movilidad Humana (OBIMID). Recuperado de https://www.documentcloud.org/documents/ 3406604-Informe-Julio-Agosto-2016.html

Saéz, R. (2006). La educación intercultural. Revista de Educación, (339), 859-881.Recuperado de: https:// dialnet.unirioja.es/servlet/articulo?codigo=2057316

Segura, L. (2016). Percepción de los docentes sobre sus alumnos migrantes en escuelas primarias de la ciudad de Rosario. Comparative Cultural Studies: European and Latin America Perspectives, (2), 15-23. doi: 10.13128/ ccselap-19994 
Stang, F., Stefoni, C. (2016). La microfísica de las fronteras. Criminalización, racialización y expulsabilidad de los migrantes colombianos en Antogasta.Astrolabio, Nueva época, (17), 42 - 80. Recuperado de: https:// revistas.unc.edu.ar/index.php/astrolabio/article/view/15781

Stefoni, C., Acosta, E., Gaymer, M., \& Casas-Cordero, F. (2008). Niños y niñas inmigrantes en Santiago de Chile. Entre la integración y la exclusión. Santiago, Chile: OIM- UAH.

Stefoni, C., Stang, F.\& Riedemann, A. (2016). Educación e interculturalidad en Chile: un marco para el análisis. Estudios Internacionales, (185), 153 - 182. doi:10.5354/0719-3769.2016.44534

Stefoni, C., Corvalán, J., Riedemann, A. y Stang, F. (2018). Informe final. Construyendo escuelas interculturales: elaboración participativa de una hoja de ruta para asistentes de la educación, profesores y directivos. Centro de Estudios MINEDUC, Fondo de Investigación y Desarrollo en Educación (FONIDE). Recuperado de: http://centroestudios.mineduc.cl/wpcontent/uploads/sites/100/2018/10/Informe-final-FONIDE-FX11622Stefoni_apDU.pdf

Stefoni, C., Stang, F. (2017) .La construcción del campo de estudio de las migraciones en Chile: notas de un ejercicio reflexivo y autocrítico. Revista Íconos, 58, 109-129.doi: 10.17141/iconos.58.2017.2477

Taylor, C. (1992). Multiculturalism and thePolitics of Recognition»: AnEssaywithCommentary. New York, USA: Princeton University Press.

Tijoux, M.E. (2013 a). Las escuelas de la inmigración en la ciudad de Santiago: elementos para una educación contra el racismo. Polis Revista Latinoamericana 12 (35), 287-307.doi:10.4067/S0718-65682013000200013

Tijoux, M.E. (2013b). Niños(as) marcados por la inmigración peruana: estigma, sufrimientos, resistencias. Convergencia,s 20 (61), 83-104. Recuperado de: http://www.scielo.org.mx/ scielo.php?script=sci_arttext\&pid=S1405-14352013000100004

Vila, R. (2003). El desarrollo de una competencia comunicativa intercultural en una sociedad multicultural y plurilingue: una propuesta de instrumentos para su evaluación. Barcelona: ICE.

Villalta, M. A. (2016). Educación intercultural en Latinoamerica: Análisis de las investigaciones de campo en la región. Psicoperspectivas, 15 (1), 130-143. doi: 10.5027/psicoperspectivas-Vol15-Issue1-fulltext-605

Walsh, C., Viaña, J., Tapia, L. (2010). Construyendo interculturalidad crítica. La Paz, Bolivia: Instituto Internacional de Integración del Convenio Andrés Bello. 